\title{
Applicability of Portable Health Clinic to Ageing Society
}

\author{
Ashir Ahmed ${ }^{1}$, Andrew Rebeiro-Hargrave ${ }^{1}$, Rafiqul Islam ${ }^{2}$, \\ Sozo Inoue ${ }^{3}$, and Naoki Nakashima ${ }^{4}$ \\ ${ }^{1}$ Faculty of Information Science and Electrical Engineering, Kyushu University \\ 744, Moto'oka, Nishi-ku, Fukuoka, Japan \\ \{ashir, andrew\}@f.ait.kyushu-u.ac.jp \\ ${ }^{2}$ Global Communicaiton Center, Grameen Communications \\ 9F, Grameen Bank Bhaban, Mirpur-2, Dhaka-1216, Bangladesh \\ rimaruf@grameen.com \\ ${ }^{3}$ Faculty of Engineering, Kyushu Institute of Technology \\ 1-1 Sensui-cho, Tobata-ku, Kita kyushu, Japan \\ sozo@mns. kyutech.ac.jp \\ ${ }^{4}$ Kyushu University Hospital, 3-1-1, Maidashi, \\ Higashi-ku Fukuoka, Japan \\ nnaoki@info.med.kyushu-u.ac.jp
}

\begin{abstract}
Portable Health Clinic is adapted to provide primary care to a super aged society. A super aged society occurs when, one third of the population is $65+$ years and one fifth is $85+$ years. The combination of aging society and incidence of non-communicable diseases increases the prevalence of elderly disability and places pressure on health care systems, health costs, and existing social norms. The goal is to reposition the Portable Health Clinic as a health information and affordable disability prevention system. In this paper, we show how the healthcare worker can supplement her competence on medical risk factors with sensor technology and share her knowledge with elderly patients within the community. We suggest that the remote telemedicine call center should be used to support distant screening and surveillance programs and provide early intervention to diseases. We investigate 18,278 Portable Health Clinic Electronic Health Records between 2012 and 2013 to see what are the most important risk factors for ill health in Bangladesh. The field data implies that elevated blood pressure and blood sugar and protein in the urine and the most important risk factors for the elderly population ( $>65$ years) when compared to younger population ( $<65$ years).
\end{abstract}

Keywords: Portable Health Clinic, Ageing Society, Personal Health Records (PHR), Remote Health Consultancy, BigData.

\section{Introduction}

The world is facing a situation without precedent: we soon will have more older people than children and more people at extreme old age than ever before. In Japan, it is expected that by 2030 , one in every three people will be $65+$ years and one in five 
people 75+ years [1]. The transition from the current technological society to a super aged society will impact on long-term health care systems, local community support systems, and change the social structure with a large percent of elderly people living alone or with a spouse.

As aging populations grow there will be a corresponding increase of lifestyle related diseases. According to the World Health Organization over the next 10 to 15 years, people in every world region will suffer more death and disability from noncommunicable diseases (NCDs) such as heart diseases, cancers, and diabetes than from infectious and parasitic diseases [2]. Lifestyle related diseases are related to unhealthy diet and physical inactivity and manifest as raised blood pressure, blood glucose, raised blood lipids and overweight and obesity. Natural aging is also cause for disease and disability: for each decade after age 55, the chance of stroke roughly doubles; half of all new cases of type 2 diabetes occur over the age of 55; and the prevalence of Arrhythmia (irregular heartbeat) tends to increase with age.

To accommodate the social cost of aging society and lifestyle diseases, there is a strong need for affordable primary care. In particular, for healthcare delivery models that places less reliance on doctors and more on other health workers. Affordability is tightly coupled with information and communication technologies that integrate primary healthcare and the curative models and make healthcare environments more accessible for older and morbid people [2].

Information Communication and Technologies (ICT) remote healthcare consultancy systems have been deployed in the global market. Mobile health has increased access to healthcare and health-related information for many unreached communities. Health consultancy over mobile phone is popular in developing countries such as Bangladesh and provides an alternative solution for partial healthcare delivery [5]. To the extent that one such service holder receives 15000 calls per day for health consultancy [6]. However, these mobile health services do not test the patient against a diagnosis process or use Electronic Health Records and this reduces the primary care impact. In addition, there is little scope to empower the local healthcare worker with the mobile phone procedures.

\section{Portable Health Clinic System}

The Portable Health Clinic (PHC) system was designed by Kyushu University and Grameen Communication's GCC (Global Communication Center) to be an affordable e-health system solution for people in unreached communities [9]. It consists of backend of data servers and a medical call center, and inexpensive front-end instances of portable briefcase consisting of medical sensors and measuring equipment. The frontend communicates with the back-end using mobile network coverage and Internet (see Figure 1).

The PHC back-end comprises GramHealth software applications, database, and medical call center. GramHealth software applications processes patient Electronic Health Records (EHR) and doctor's e prescriptions, and stores in a database. Doctors at the medical call center access GramHealth database through the Internet or 
have a copy in a local server. Upon receiving a multimedia call from a patient, the doctor can find patient's previous EHR, can create, and send an e-prescription [10]. This saves time and effort as the doctor does not need to questions about the patients' personal profile (basic attributes and medical history) but can focus on the immediate health inquiry.

The PHC front-end consists of a brief case containing medical sensor devices developed with international information standards, data transmission system with mobile network, data management application, telemedicine by Skype connection, and local Electronic health record preservation. The PHC briefcase is meant to be low cost and portable. It is envisaged to cost less than US\$300 and can be carried by a female healthcare worker. The PHC briefcase will be owned and operated by a community health entrepreneur. This will be sustainable business model as the Health entrepreneur can build a professional relationship with her local cliental [11].

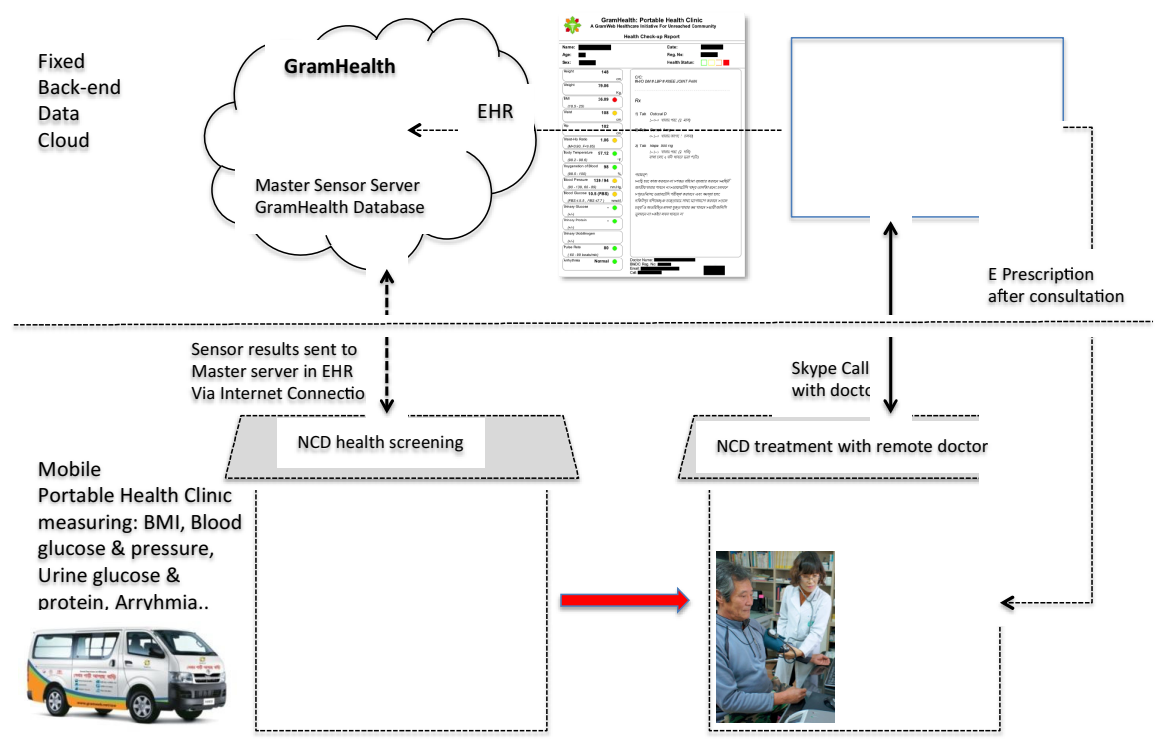

Fig. 1. Portable Health Clinic system architecture: back end data center and front ends health briefcases containing a comprehensive health measuring system that are be carried to a person house or community center.

\section{Empowering the Healthcare Worker}

The healthcare worker is the point of contact between the Portable Health Clinic service and the elderly patient health needs. The healthcare worker personally interacts with the elderly patient and can provide a social/community connection to socially isolated elderly persons (living alone). The Portable Health Clinic empowers the healthcare: she is responsible for large array of medical tests; she is in control of the sensors and devices; and she can explain the triage logic, and sets up the telemedicine call to the remote medical doctor (Table 1). 
Table 1. Portable Heath Clinic tool kit

\begin{tabular}{|l|l|l|l|l|}
\hline $\begin{array}{l}\text { Personal } \\
\text { Data }\end{array}$ & $\begin{array}{l}\text { Personal } \\
\text { Characteristics }\end{array}$ & Risk Factors & $\begin{array}{l}\text { Medical Indica- } \\
\text { tors }\end{array}$ & Intervention \\
\hline Sex & Height $(\mathrm{cm})$ & Body Temperature & Urine Sugar & Color Status \\
\hline Age & Weight $(\mathrm{kg})$ & Blood Sugar & Urine Protein & $\begin{array}{l}\text { prescrip- } \\
\text { tion_id }\end{array}$ \\
\hline Address & BMI & PBS/FBS & $\begin{array}{l}\text { Urine Urobilino- } \\
\text { gen }\end{array}$ & Site ID \\
\hline Mobile No. & Waist & Blood Hemoglobin & SpO2 & \\
\hline Phase ID & Hip & Blood Pressure(sys) & Pulse Ratio & \\
\hline $\begin{array}{l}\text { Check-up } \\
\text { ID }\end{array}$ & W/H Ratio & Blood Pressure(dia) & Arrythmia & \\
\hline $\begin{array}{l}\text { Check-up } \\
\text { Date }\end{array}$ & & & & \\
\hline
\end{tabular}

\subsection{Briefcase Sensors and Materials}

The healthcare worker "s toolkit is a light-weight briefcase consists of "easy-to-use" inexpensive sensors based on international information standards that use either standard transmission or 'Body Area Network' (BAN) interface on the sensor (Table 2). The briefcase also includes tablet PC, or note PC, consumable goods such as urine tester tapes and blood sugar cubes, battery unit and dry cells, a measure, a barcode scanner, papers for writing and printing. The total weight of the case is about 10 kilos including the weight of the briefcase [12].

Table 2. Sensor and equipment for a typical Portable Health Clinic briefcase

\begin{tabular}{|l|l|l|l|l|}
\hline Sensor & Maker & Product Code & Transmission & $\begin{array}{l}\text { Weight } \\
\text { (gms) }\end{array}$ \\
\hline Weight Scale & A\&D & UC-321PBT & BAN & 2500 \\
\hline Blood Pressure & A\&D & UC-321PBT & BAN & 300 \\
\hline Pulse Oxymeter & OXiM & S-101 & BAN & 60 \\
\hline Blood Glucose & Terumo & MEDISAFEFIT & Felica & 50 \\
\hline Body Temperature & Terumo & W520DZ & Felica & 27 \\
\hline FeliCa Reader & Sony & PaSoRi & USB & 35 \\
\hline Mobile Printer & $\begin{array}{l}\text { Hewlett } \\
\text { Packard }\end{array}$ & OfficeJet 100 & Bluetooth & 2500 \\
\hline Mobile Scanner & Fujitsu & FI-S1100 & USB & 350 \\
\hline Web Camera & Logicool & HD & & \\
\hline
\end{tabular}




\subsection{Sensor System Architecture}

The healthcare worker samples the elderly patient's risk factors and medical indicators using sensors and devices during a 15-minute health checkup. A local sensor server (within the briefcase) receives and stores patient data via wireless-LAN and synchronizes its cache with the master sensor server when an Internet connection is available. The master sensor server in the back-end data cloud stores all sensor data and provides data to the Personal Health Record Server and doctors in the call center (see Figure 1). The interface of the local sensor server is the same of that of the master sensor server; therefore sensor boxes can directly connect to the master sensor server by changing the configuration address.

\subsection{Morbidity Stratification Algorithm}

The healthcare worker will inform the elderly patient that risk of morbidity (poor health) is identified using a triage stratification algorithm in the local sensor server. The elderly patient's risk factor measures of each health check-up item are compared against risk stratification matrix based on International diagnosis standards (WHO). The algorithm categorizes and grades the sensor results according to a triage: green (healthy), yellow (caution), orange (affected), and red (emergency). The current triage risk stratification is parameterized against a "B-logic" (Bangladesh logic) and an example of the categories is shown in Figure 2. The individual health condition can be enhanced by integrating results of a questionnaire into the 4 degrees by the worst color of all health check-up items.

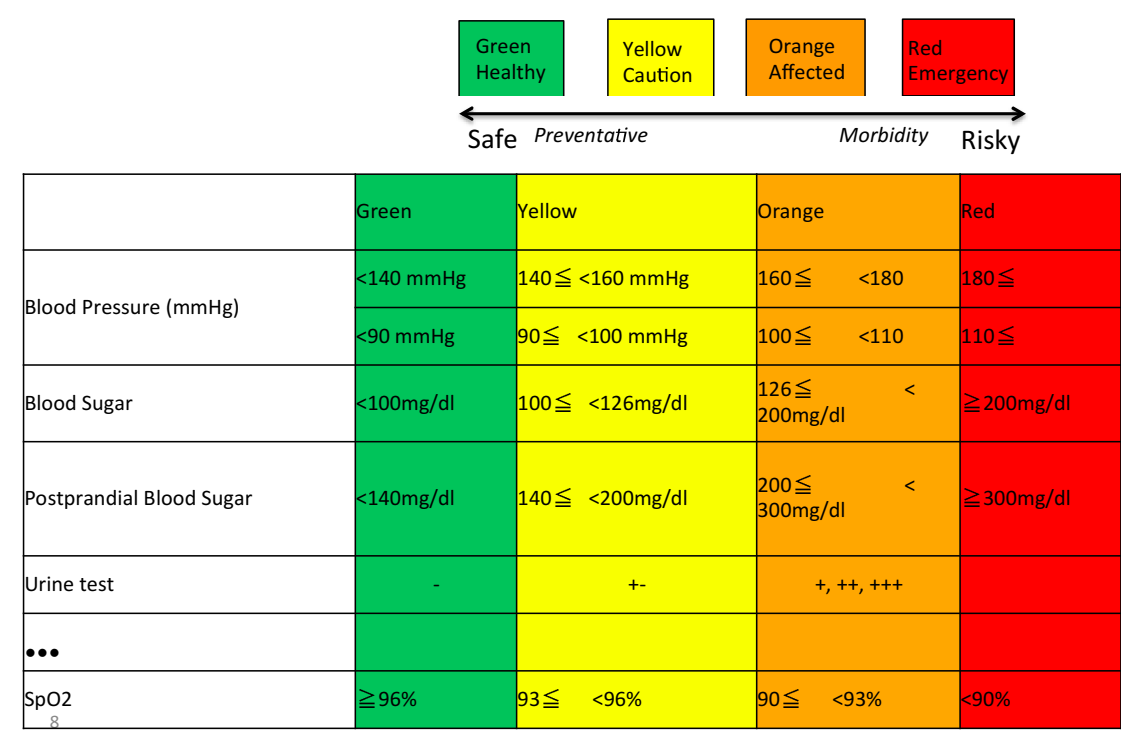

Fig. 2. Triage categories are based on medical recommendations 


\subsection{Booklet for Health Guidance}

The healthcare worker gives the elderly patient a 11-page booklet that describes the advantages of a controlled diet and regular physical exercise and disadvantages of obesity, smoking, and chronic diseases such as hypertension, diabetes, and kidney disease.

\subsection{Telemedicine and e-Prescription}

The healthcare worker sets up a telemedicine session for orange (affected) and red (emergency) subjects using mobile network coverage to connect the patient to the medical call center at the back- end. In the call center, male and female doctors are available to provide telemedicine consultancy. Doctors access the electronic results of the patient's health check-up and provide advice for the disease or condition and can issue a e-prescription for the patient to access medicine via the network.

\section{$4 \quad$ Healthcare Delivery Models}

Portable Health Clinic is a healthcare delivery model that provides affordable primary care to the aging society. At it core, the Portable Health Clinic system is an ICT solution that informs the elderly person his or her health status. This is very important service, as most people are unsure of their own health status. For example, 35 to 44 per cent of people with diabetes do know they have it. Diabetes symptoms often do not show up until several years after the onset of the disease. PHC screening for high blood glucose levels and allowing for timely intervention can reduce disability of a large proportion of the aging society. However, mass screening is not practical for the PHC even though it fits in a briefcase. It is more feasible to create a business model where local community healthcare worker or an individual takes responsible of the patient registration and clinical measurements and GramHealth provides healthcare guidelines, medical doctor call center, and EHR database (Figure 3). Allowing a community healthcare worker or family individuals to take ownership of health measurements localizes medical health care.

The Portable Health Clinic is used for medical screening (group checking) or as a tool for medical surveillance (monitoring individuals at home) (Figure 3). The purpose of the medical screening is to identify disease early and enable earlier intervention and management in order to reduce the mortality and suffering from the disease. The purpose of medical surveillance is to follow-up on high-risk patients (identified as orange and red) and periodically measures the effects of exposure to lifestyle and environmental toxins on their condition. The PHC surveillance programs includes the following:

1. Baseline screening by Portable Health Clinic to establish medication condition;

2. Periodic visits by the Portable Health Clinic to evaluate the level of intervention;

3. Termination and handover to health system when the patient is chronically ill. 
In each step, a doctor will available at the call center to evaluate the results, discuss the conditions with the patient, make recommendations, and send e prescriptions to the patient.

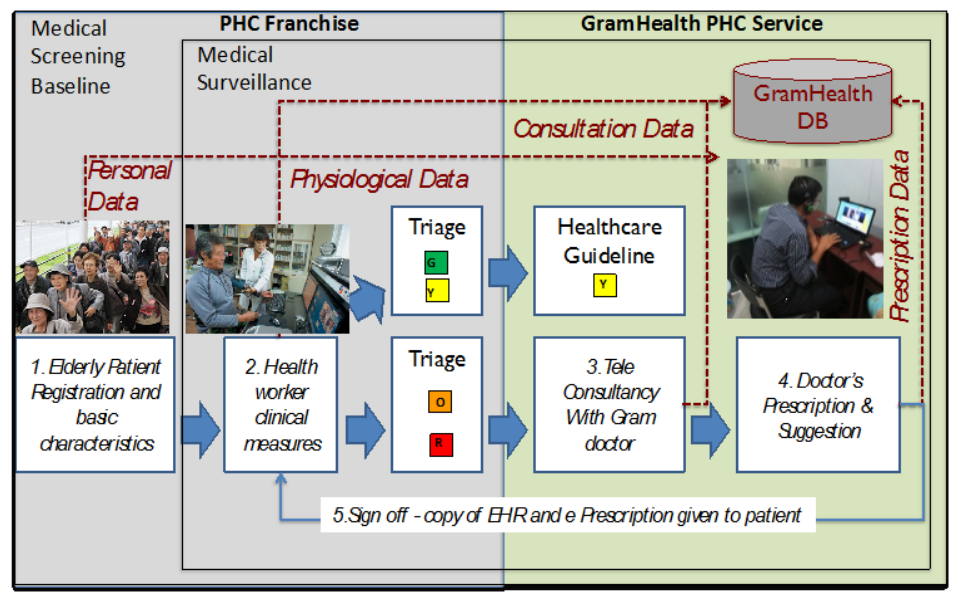

Fig. 3. Healthcare delivery models: group screening for early disease identification and home surveillance for elderly patient with a health risk

\section{$5 \quad$ Identifying the Most Important Risk Factors in Aging Society}

The Portable Health Clinic currently identifies 14 risk factors associated with heart attacks, strokes, diabetes and other conditions (Table 2). For intervention policy, it is necessary to establish which are the most important risk factors in an aging society. This is achieved by analyzing the PHC data collected from Bangladesh.

Portable Health Clinic was tested against as random populations living in unreached communities in Bangladesh. The test subjects were volunteers that responded to awareness campaigns conducted in urban, sub-urban and rural areas between September 2012 and November 2013. The experiment environment consists of the following facilities:

(a) Small call center in Dhaka (the capital city of Bangladesh) with two female and two male doctors, and one transcript writer;

(b) A portable health clinic briefcase with 12 diagnostic tools;

(c) Mobile health check-up team consisting two health assistants, 3 program assistants and one quality check officer. Patient data was captured from diagnostic tools wireless BAN and by manually inserted into our GramHealth database through a user-friendly web interface;

(d) Off-line version of GramHealth to store the patient's health profile and synchronize with the central server when the sufficient network bandwidth is available;

(e) On-line GramHealth software tools to process and store patient electronic health records. 
The mobile health team visited rural villages and urban factories, and set up a PHC health camp service for one week (Figure 4). There was a marketing campaign 2 weeks in advance so that subjects could preregister to the event and book a time for a health checkup. The health team revisited the areas every two months to repeat the measures. Patients who were previously measured as risky (orange and red) were asked to join the next health camp for a new check-up.
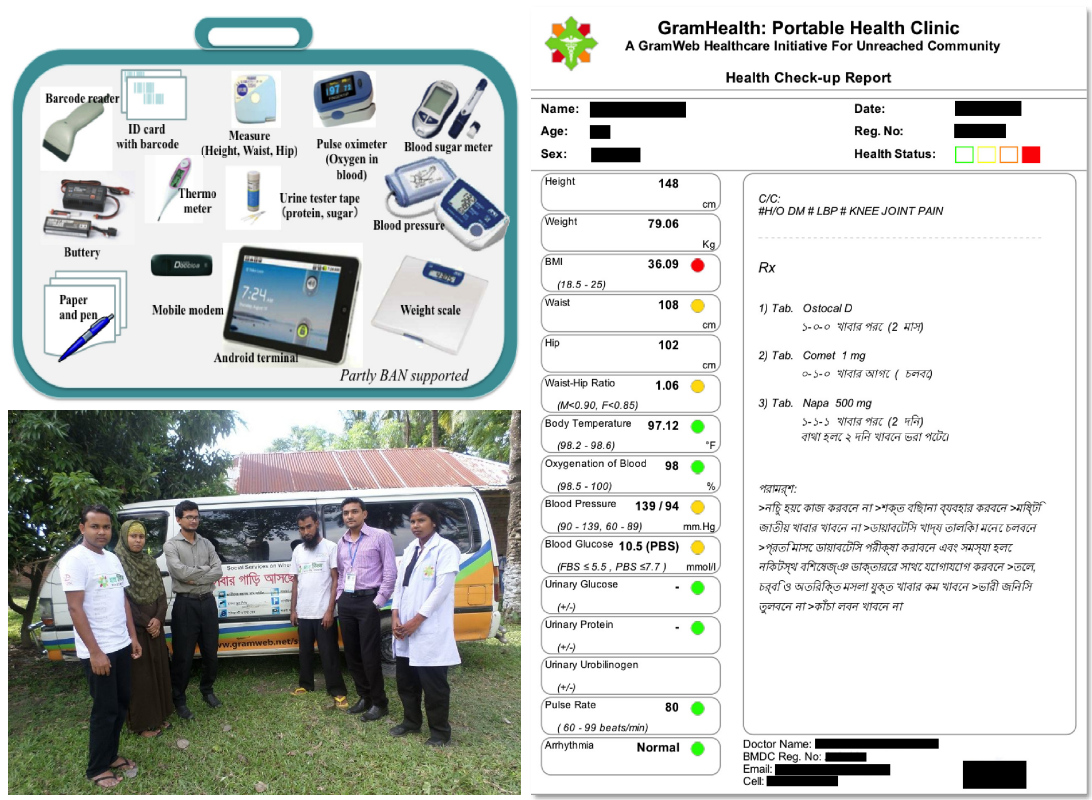

Fig. 4. Portable Health Clinic local team, the sensors used and Electronic Health Recorde generated during the experiment

During the experimental time frame, 18,278 patients received a health check and their EHRs are stored in the GramHealth database. The distribution between young and old is 17,252 less 65 years (younger cohort) and 1,026 greater then 65 years (elder cohort). The oldest patient was 106 years. The experiment was carried out in six rural regions and three urban areas including Dhaka. In one rural region, there is no mobile network coverage and the offline GramHealth(local sensor server) perfectly analyzed the data and classified patients into four groups. Telemedicine session with the remote doctor from this area point was not possible.

\section{Comparing Elderly Cohort Data Against Young Cohort Data}

The Portable Health Clinic identified morbidity for a large number of patients who were unaware of their illhealth. During the health checkup, 18,278 subjects were tested. PHC categorized 11,992 patients as low risk (Green 1,976 and Yellow 
10,016) and 6,276 patients as high risk (Orange (affected) 5,435 and Red (emergency) 841). There were 6,276 patient-doctor telemedicine consultations. The high-risk patients were prescribed medicine and were informed to attend the next PHC health care camp for a follow-up health check-up, scheduled 2 months later. When comparing the young/old division, the elder cohort (greater than 65 years) was twice as likely to be categorized as high-risk patients than the younger cohort (less than 65 years). Of all the elderly tested, $60.1 \%$ had a telemedicine session and received treatment against $33 \%$ of the sample less than 65 years old (Table 3). In particular, the elder cohort was three times more likely to be categorized as an emergency case.

Table 3. Distribution of Triage results

\begin{tabular}{|l|l|l|}
\hline Triage Categories & $<65$ younger & $>65$ Elder \\
\hline Green (healthy) & $11.3 \%$ & $2.5 \%$ \\
\hline Yellow (caution) & $55.8 \%$ & $37.9 \%$ \\
\hline Orange (affected) & $28.7 \%$ & $47 \%$ \\
\hline Red (emergency) & $4.09 \%$ & $13.1 \%$ \\
\hline
\end{tabular}

The general patient physical characteristic of the sampling frame reveals low BMI data for both younger cohort and the elder cohort. The experiment was conducted in rural Bangladesh villages and it appears that BMI is not currently an issue. Low BMI indicates that in this test, high blood glucose and blood pressure and are not related to overweight or obesity. There is a marked difference between patients suffering from Arrythmia (irregular heartbeat). The data shows that this is mainly a problem for the elder cohort (Table. 4.)

Table 4. Physical characteristics results

\begin{tabular}{|l|l|l|l|l|}
\hline $\mathrm{N}=18,278$ & \multicolumn{2}{|l|}{ BMI } & \multicolumn{2}{l|}{ Arrythmia } \\
\hline & $<65$ & $>65$ Elderly & $<65$ & $>65$ Elderly \\
\hline Orange (affected) & $0.4 \%$ & $0.4 \%$ & $0.23 \%$ & $6.4 \%$ \\
\hline Red (emergency) & $0.05 \%$ & $0.3 \%$ & & \\
\hline Total & $0.45 \%$ & $0.7 \%$ & $0.23 \%$ & $6.4 \%$ \\
\hline
\end{tabular}

The results of the blood tests of the sampling frame show significant differences between young cohort and elder cohort. Elderly patients were portrayed with high blood sugar levels inferring a greater propensity of developing diabetes II than the young cohort. Elderly patients were characterized with high blood pressure suggesting a significantly higher risk of stroke or heart attack compared to the younger cohort (Table 5). There are little differences in the SP02 measures between cohorts and this implies that blood oxygen is not an important risk factor in Bangladesh. 
Table 5. Blood sample results showing increased risk for aging population

\begin{tabular}{|l|l|l|l|l|l|l|}
\hline Blood Samples & \multicolumn{2}{l|}{ High Blood Sugar } & \multicolumn{2}{l|}{ High Blood Pressure } & \multicolumn{2}{l|}{ Sp02 } \\
\hline & $<65$ & $>65$ Elderly & $<65$ & $>65$ Elderly & $<65$ & $>65$ Elderly \\
\hline Orange (affected) & $0.4 \%$ & $13.25 \%$ & $0.017 \%$ & $12.37 \%$ & $0.15 \%$ & $0.29 \%$ \\
\hline Red (emergency) & $0.15 \%$ & $5.5 \%$ & $0.008 \%$ & $8.77 \%$ & $0.08 \%$ & $0.58 \%$ \\
\hline Total & $0.19 \%$ & $18.75 \%$ & $0.0026 \%$ & $21.15 \%$ & $0.23 \%$ & $0.87 \%$ \\
\hline
\end{tabular}

The results of the urine tests of the sampling frame show noticeable differences between young cohort and elder cohort. High blood sugar in urine combined with high blood glucose indicates that $9 \%$ of the elders screened have developed diabetes II. High protein in urine is an indicator for heavy metal poisoning. The PHC data has captured the effects of arsenic contamination, and it appears the elder cohort have been exposed to groundwater arsenic for longer than the younger cohort. Urobilinogen is an indicator for liver disease and does not appear to be a high risk factor.

Table 6. Urine sample results shows increased risk for aging population

\begin{tabular}{|l|l|l|l|l|l|l|}
\hline Urine Samples & \multicolumn{2}{l|}{ Sugar } & \multicolumn{2}{l|}{ Protein } & \multicolumn{2}{l|}{ Urobilinogen } \\
\hline Orange (affected) & $<65$ & $>65$ Elderly & $<65$ & $>65$ Elderly & $<65$ & $>65$ Elderly \\
\hline Low Risk + & $2.08 \%$ & $2.8 \%$ & $5.65 \%$ & $13.06 \%$ & $0.25 \%$ & $1.85 \%$ \\
\hline Medium Risk ++ & $2.6 \%$ & $2.14 \%$ & $3.14 \%$ & $4.77 \%$ & $0.046 \%$ & 0 \\
\hline High Risk +++ & $2.49 \%$ & $3.5 \%$ & $0.37 \%$ & $0.9 \%$ & $0.005 \%$ & 0 \\
\hline Total & $6.38 \%$ & $8.47 \%$ & $9.16 \%$ & $18.85 \%$ & $0.3 \%$ & $1.85 \%$ \\
\hline
\end{tabular}

\section{Discussion}

The Portable Health Clinic is an affordable and efficient E health system that identifies morbidity in unreached communities. It's compact form, allows a healthcare worker to take the briefcase of sensors to any location or disaster area at short notice. The sensors and devices are easy-to-use, to the extent that that the healthcare worker can provide her local community a high-tech primary care. The back-end of servers, operated by Gramhealth can provide group medical screening and personal medical surveillance services to any community. The PHC system has been validated in Bangladesh. The system recognized diseases for 6,276 patients, of which 846 were emergencies (lives saved). Prior to their PHC measurements, the patients were apparently feeling in good health and were unaware of the their risk factor or they were feeling unwell but the disease was not diagnosed. The results show that elder cohort $(>65)$ were at more risk than the younger sample. This is due to natural ageing process and longer exposure to life style disease. The most important risk factors were: blood pressure, blood glucose and protein in urine. These are followed by sugar in urine and Arryhmia. 
These are good results, however there are constraints to the Portable Health Care system concerning scale, adaptability and interoperability. The portable health clinic is a mobile small-scale solution and cannot be compared to a large multifunctional health system such as a hospital. It can treat 1000s of patients and not millions of patients. The current PHC briefcase contains sensors that measure non-communicable morbidity and not infectious diseases or dementia. The Electronic Health Records are not interoperable with other E Health systems and the EHR stays within the GramHeath database until there is a standardized EHR format. Accepting these limitations, the Portable Health Clinic does provide 'on the ground' practical heath care for an aging society: low-income populations, and for short-term catastrophe coverage. It is based on a sustainable business model where the healthcare workers are localized (semi skilled entrepreneur and Health Assistant) and the experts consultants (doctors) do not travel or move to undesirable areas.

\section{Conclusion and Future Works}

In this work, we introduced the Portable Health Clinic system. We explained the system components, and operations. We created an experimental design and tested it against a large sample population in Bangladesh. Results showed that PHC could be used to identify morbidity and help reduce morbidity by providing health consultancy in unreached areas.

\section{References}

1. Muramatsu, N., Akiyama, H.: Japan: Super-Aging Society Preparing for the Future. The Gerontologist 51(4), 425-432

2. Global Health Issues, http://www.globalissues.org/issue/587/healthissues (accessed on March 1, 2013)

3. The Remote and Rural Steering Group.: Delivering for Remote and Rural Healthcare. The Scottish government, Edinburg (November 30, 2007)

4. Chaudhury, N., Hammer, J., Kremer, M., Muralidharan, K., Rogers, F.H.: Missing in Action: Teacher and Health Worker Absence in Developing Countries. The Journal of Economic Perspectives 20(1), 91-116 (2006)

5. Ahmed, A., Osugi, T.: ICT to change BOP: Case Study: Bangladesh. Shukosha, Fukuoka, 139-155 (November 2009)

6. Kai, E., Ahmed, A.: Technical Challenges in Providing Remote Health Consultancy Services for the Unreached Community. In: Proceeding of 27th IEEE International Conference (AINA), FINA-2013 Workshop, Barcelona, Spain (March 2013)

7. Chase, H.P., Pearson, J.A., Wightman, C., Roberts, M.D., Oderberg, A.D., Garg, S.K.: Modem Transmission of Glucose Values Reduces the Costs and Need for Clinic Visits. Diabetes Care 26(5), 1475-1479 (2003)

8. Bayliss, E., Steiner, J.F., Fernald, D.H., Crane, L.A., Main, D.S.: Descriptions of barriers to self-care by persons with comorbid chronic diseases. Ann. Fam. Med. 1(1), 15-21 (2003) 
9. Cafazzo, J.A., Leonard, K., Easty, A.C., Rossos, P.G., Chan, C.T.: Bridging the self-care deficit gap: Remote patient monitoring and hospital at home. In: Electronic Healthcare First International Conference, eHealth (2008) (February 14, 2009)

10. Kato, S.: A Study on Implementing a Portable Clinic based on Social Needs. Undergraduate Thesis, Kyushu University (March 2012)

11. Nessa, A., Ameen, M., Ullah, S., Kwak, K.: Applicability of Telemedicine in Bangladesh: Current Status and Future Prospects. The International Arab Journal of Information Technology 7, 138-145 (2010)

12. Ahmed, A., Ishida, K., Okada, M., Yasuura, H.: Poor-Friendly Technology Initiative in Japan: Grameen Technology Lab. The Journal of Social Business 1(1) (January 2011)

13. Naoki, N., Nohara, Y., Ahmed, A., Kuroda, I.S., Ghosh, P., Islam, R., Hiramatsu, T., Kobayashi, K., Inoguchi, T., Kitsuregawa, M.: An Affordable, Usable and Sustainable Preventive Healthcare System for Unreached People in Bangladesh. Report (2013)

14. Kai, E., Ahmed, A.: Remote health consultancy service for unreached community: Amazing facts and technical challenges. In: Proceedings of the First MJIIT-JUC Joint Symposium, MJIIT, UTM, Kulalumpur, Malaysia, November 21-23 (2012) 